\title{
Functional outcome after one-stage flap reconstruction of the hypopharynx following tumor ablation
}

\author{
Talisa D. van Brederode ${ }^{1} \cdot$ Gyorgy B. Halmos $^{2} \cdot$ Martin W. Stenekes $^{1}$
}

Received: 3 June 2016/Accepted: 19 August 2016/Published online: 26 August 2016

(c) The Author(s) 2016. This article is published with open access at Springerlink.com

\begin{abstract}
The main objective of this study was to evaluate functional outcome in terms of food passage of the three different reconstruction techniques that are currently most often used for hypopharyngeal reconstruction in our institution. A retrospective observational database research was conducted of all patients that underwent hypopharyngeal reconstruction for carcinoma of the hypopharynx or larynx from 1992 until 2014 in the University Medical Center Groningen. The following techniques were most commonly used and therefore analyzed: the pedicled pectoralis major flap, the radial forearm free flap and the anterolateral thigh free flap. Our primary outcome food passage was measured after 1 year and classified in gastric tube fed, fluids, semisolid or solid. Complications were registered according to the Clavien Dindo classification in five different grades. Comorbidity was scored using the Adult Comorbidity Evaluation Index. 58 patients were included. 51 patients survived one year follow up, $25 \%$ returned to a solid diet, $40 \%$ returned to a semi-solid diet and $20 \%$ remained feeding tube dependent. Overall flap success rate was 88 and $35 \%$ developed a pharyngocutaneous fistula. Multivariable ordinal regression showed that reconstruction with free flaps, a near-circumferential surgical defect, a higher body mass index and no comorbidity showed significantly better functional outcomes in the food passage. For recipient site
\end{abstract}

Martin W. Stenekes

m.w.stenekes@umcg.nl

1 Department of Plastic Surgery, University of Groningen, University Medical Center, Groningen, Postbus 30.001, 9700 RB Groningen, The Netherlands

2 Department of Otorhinolaryngology, Head and Neck Surgery, University of Groningen, University Medical Center Groningen, Groningen, The Netherlands complications, both free flaps and a shorter surgery time resulted in less severe complications. This study shows that the use of free flaps is superior to the use of the pectoralis major flap, and that it should therefore be reserved as a second choice.

Keywords Reconstruction · Free flaps · Pectoralis major · Hypopharynx · Interposition flap

\section{Introduction}

Surgery of advanced laryngeal and hypopharyngeal carcinoma may include partial or total laryngectomy with or without partial or total pharyngectomy. This will potentially leave large, sometimes circumferential defects which require complex reconstruction $[1,2]$.

There are many factors that contribute to the choice of a certain reconstruction technique. For instance, the size and level of the defect, patient's general medical health and wishes, the surgeon's experience and preference of different techniques are all factors to be taken into consideration. The transferred tissue needs to withstand high dose radiation therapy (usually adjuvant radiotherapy is required), not bulky and pliable so it can cover a circumferential defect and preferably not be too close to the neck so that two teams can operate simultaneously [3].

Many reconstruction techniques have been developed and successfully used. Myocutaneous pedicled flaps (pectoralis major, deltopectoral, latissimus dorsi), visceral transposition (jejunal autograft, colon autograft, gastric pull-up), free fasciocutaneous flaps (anterolateral thigh, radial forearm, scapular) and more have been described in literature [4]. As regarding to complication rates and 
functionally in swallowing and speech, each technique has its advantages and limitations.

The surgical defect that arises in the hypopharynx after tumor ablation can be circumferential, near-circumferential or partial (the latter is left out of consideration in this study). Sometimes it is oncologically safe to leave a mucosal strip of the posterior pharyngeal wall intact. This means that the reconstruction flap is sutured to the posterior pharyngeal wall, creating two additional vertical suture lines. In theory this could be a potential point of weakness, leading to dehiscence of the flap and pharyngocutaneous fistulas. On the other hand, some experts suggest that a near-circumferential defect creates less long-term strictures [1].

In our institution we generally use three types of hypopharyngeal reconstruction, namely the anterolateral thigh free flap (ALTFF), the radial forearm free flap (RFFF) and the pedicled pectoralis major myocutaneous flap (PMMF). The PMMF is often too bulky, making it aesthetically and functionally non-satisfying. Therefore, the PMMF is preserved as a second choice in our institution, for high risk patients in whom a free vascularized fasciocutaneous flap has failed or is considered too risky. The RFFF and ALTFF are both considered to have good results, although the ALTFF cannot be used in obese patients.

Currently there is no consensus on the primary preference for hypopharyngeal reconstruction. The objective of the present study is to evaluate functional outcome viz. voice rehabilitation and food passage of the three different reconstruction techniques that are currently used in this institute.

\section{Materials and methods}

In this retrospective observational database study, clinical records of all patients that underwent hypopharyngeal reconstruction for carcinoma of the hypopharynx or larynx between 1992 and 2014 in the University Medical Centre Groningen were collected. These defects were reconstructed using RFFF, ALTFF or PMMF.

\section{Clinical variables}

Patient and treatment information was obtained from electronic patient dossiers. The following variables were included: age, sex, tumor site, cancer stage, preoperative body mass index (BMI), postoperative BMI at discharge, the American Society of Anesthesiologists (ASA)-score, pre- and postoperative therapy (such as chemotherapy or radiotherapy), any history of malignancy, length of the surgery, type of reconstruction, surgical defect, the number of hospital days and postoperative complains such as dysphagia, reflux and aspiration. The Adult Comorbidity Evaluation 27 (ACE-27) index was used to score preoperative comorbidity [5].

\section{Outcome measures}

Functionality in food passage after full recovery was classified in the following outcome categories: gastric tube fed fluids, fluids, semisolid (pureed soft food) or solid. This information was preferably retrieved from a dietician report, approximately one year postoperatively.

Speech function after reconstruction was also evaluated. The type of speech used was classified in: electrolarynx, esophageal speech or tracheoesophageal puncture and prosthesis. The quality and patient satisfaction of speech were also evaluated.

Complications were registered according to the Clavien Dindo classification in five different grades [6, 7]. These complications were classified as related to the recipient site, donor site and medical complications [8, 9]. Clinically relevant complications were also listed separately, such as flap necrosis and pharyngocutaneous fistulas.

\section{Statistical analysis}

For statistical analysis IBM SPSS Statistics 22.0 was used [10]. Descriptive statistics was used to display patient characteristics, treatment details and functional outcome. To evaluate significant factors that affect the food passage and recipient site complications, multivariable ordinal regression was used.

There are probably more factors contributing to both the choice of flap reconstruction and the functional outcome food passage. Based on univariable ordinal regression (with $\alpha=15 \%$ [11]), expert opinion and literature, five variables were identified for multivariable ordinal regression analysis. These variables were: type of reconstruction (PMMF, ALTFF, RFFF), surgical defect (circumferential or near-circumferential), ACE-27 (grade III, grade II, grade I, none), postoperative radiotherapy (yes or no) and preoperative BMI (continuous variable).

Recipient site complications is also an ordinal variable, classified in grades of severity from no complication at all to grade $\mathrm{V}$, meaning death. In all likelihood, more factors than just the type of reconstruction used will have an impact on recipient site complications. In a similar way as described above, five variables were selected to participate in the multivariable ordinal regression model. These variables were: type of reconstruction (PMMF, ALTFF, RFFF), surgical defect (circumferential or near-circumferential), ACE-27 (grade III, grade II, grade I, none), length 
of the surgery (scale variable, defined in minutes) and preoperative radiotherapy (yes or no).

\section{Results}

\section{Demographics}

In total 58 patients were included in this study. The median age was 62 years, and most patients were men (male:female ratio: 4.27:1). Median BMI was $23.3 \mathrm{~kg} / \mathrm{m}^{2}$. Most patients had mild comorbidity according to the ACE-27 index. Over half of the patients have had previous radiotherapy in the head and neck area. 18 patients had already undergone total laryngectomy, after which the cancer had recurred or a second primary tumor developed, needing further resection and therefore reconstruction. For further demographic details, see Table 1.

\section{Surgical outcome}

There were almost as many circumferential as near-circumferential hypopharyngeal defects (respectively, 32 and 26). In total 16 pectoralis major flaps, 11 anterolateral thigh flaps and 31 radial forearm flaps were used. Median length of laryngectomy and reconstruction was 657 minutes, ranging from 267 until 1128 minutes. Three patients died within one month after surgery, which leads to an early mortality rate of $5.2 \%$ in this population. Two of these patients died because of medical complications after major surgery (e.g. congestive heart failure and/or untreatable electrolyte disturbances leading to multiple organ failure). One patient died because of major untreatable recipient site complication. Patients were hospitalized for an average of 27 days. Over half of the patients received additional treatment in the form of radiotherapy or chemoradiation. For more treatment details, see Table 2.

\section{Functional outcome}

52 patients survived the one year follow up period, but one was excluded for functional outcome data because a jejunum flap was used to restore the pharynx after total necrosis of the original flap. Of these 51 patients, about $80 \%$ was able to be self-sufficient in oral nutrition. Almost $20 \%$ was not able to swallow at all, and was therefore feeding tube dependent. Median BMI one year postoperative was $23.2 \mathrm{~kg} / \mathrm{m}^{2}, 0.1 \mathrm{~kg} / \mathrm{m}^{2}$ lower than preoperative.

Strictures that needed endoscopic dilatation occurred in $38 \%$ of patients. Patients required a median of four dilatations each in their follow up period, ranging from 1 up to 70. Most patients needed two till four dilatations with good functional results. One patient required a monthly
Table 1 Preoperative characteristics

\begin{tabular}{|c|c|c|}
\hline$N=58$ & $n(\%)$ & Median (range) \\
\hline Age (years) & & $62(43-88)$ \\
\hline \multicolumn{3}{|l|}{ Sex } \\
\hline Male & $47(81.0)$ & \\
\hline Female & $11(19.0)$ & \\
\hline BMI $\left(\mathrm{kg} / \mathrm{m}^{2}\right)$ & & $23.3(14.4-37.1)$ \\
\hline \multicolumn{3}{|l|}{ ASA-classification } \\
\hline ASA 1 & $3(5.2)$ & \\
\hline ASA 2 & $22(37.9)$ & \\
\hline ASA 3 & $26(44.8)$ & \\
\hline ASA 4 & $2(3.4)$ & \\
\hline N/A & $5(8.6)$ & \\
\hline \multicolumn{3}{|c|}{ Pre-operative co-morbidity (ACE-27) } \\
\hline None & $16(27.6)$ & \\
\hline Mild & $28(48.3)$ & \\
\hline Moderate & $13(22.4)$ & \\
\hline Severe & $1(1.7)$ & \\
\hline \multicolumn{3}{|l|}{ Primary tumor site } \\
\hline Hypopharynx & $39(67.2)$ & \\
\hline Larynx & $19(32.8)$ & \\
\hline \multicolumn{3}{|l|}{ Cancer stage } \\
\hline Stage III & $3(5.2)$ & \\
\hline Stage IV & $24(41.4)$ & \\
\hline Recurrent cancer & $27(46.6)$ & \\
\hline N/A & $4(6.8)$ & \\
\hline History of malignancy & $21(36.2)$ & \\
\hline Head and Neck & $18(31.0)$ & \\
\hline Lung & $2(3.4)$ & \\
\hline Other & $4(6.9)$ & \\
\hline \multicolumn{3}{|l|}{ Previous treatment } \\
\hline None & $22(37.9)$ & \\
\hline (Chemo)radiation & $36(62.1)$ & \\
\hline Total laryngectomy & $18(31.0)$ & \\
\hline
\end{tabular}

dilatation but kept having good results, so that he has had a total of 70 dilatations now. The number of dilatations was not dependent on years of follow up.

About $75 \%$ of the patients were able to communicate with the tracheoesophageal puncture (TEP) and prosthesis, and about $50 \%$ of patients considered their speech as medium to good. Over $50 \%$ of patients were satisfied with the reconstruction results, with mild complaints. $37 \%$ of the patients complained of dysphagia postoperatively. For more details in functional outcome, see Table 3.

Food passage was compared between the different types of reconstruction. The pectoralis major pedicled flap had the highest percentage of feeding tube dependent patients $(35.3 \%)$, whereas only $11.8 \%$ of free flaps were tube dependent one year postoperatively. In the pectoralis major pedicled group, only $17.6 \%$ of patients regained a fully 
Table 2 Treatment details

\begin{tabular}{lcc}
\hline$N=58$ & $n(\%)$ & Median (range) \\
\hline Surgical hypopharynx defect & \\
Circumferential & $32(55.2)$ & \\
Near-circumferential & $26(44.8)$ \\
Type of reconstruction & & \\
Pectoralis Major & $16(27.6)$ \\
$\quad$ Circumferential & $4(6.9)$ \\
$\quad$ Near-circumferential & $12(20.7)$ \\
Anterolateral thigh & $11(19.0)$ \\
$\quad$ Circumferential & $10(17.2)$ \\
$\quad$ Near-circumferential & $1(1.7)$ \\
Radial forearm & $31(53.4)$ \\
Circumferential & $18(31.0)$ \\
Near-circumferential & $13(22.4)$ \\
Length of surgery (minutes) & \\
Hospital stay (days) & \\
Postoperative treatment & \\
None & \\
Chemo(radiation) & $22(37.9)$ \\
\hline
\end{tabular}

${ }^{a}$ Length of surgery includes total laryngectomy and reconstruction

Table 3 Functional outcome

\begin{tabular}{|c|c|c|}
\hline$n=51$ & $n(\%)$ & Median (range) \\
\hline BMI $\left(\mathrm{kg} / \mathrm{m}^{2}\right)$ & & $23.2(16.3-35.9)$ \\
\hline \multicolumn{3}{|l|}{ Food passage } \\
\hline Solid & $14(27.5)$ & \\
\hline Semisolid & $21(41.2)$ & \\
\hline Fluids & $6(11.8)$ & \\
\hline Gastric tube/PEG & $10(19.6)$ & \\
\hline \multicolumn{3}{|c|}{ Postoperative complaints } \\
\hline Dysphagia & $19(37.5)$ & \\
\hline Reflux & $3(5.9)$ & \\
\hline Aspiration & $4(7.8)$ & \\
\hline \multicolumn{3}{|l|}{ Speech type } \\
\hline Electrolarynx & $3(5.9)$ & \\
\hline Esophageal speech & $5(9.8)$ & \\
\hline TEP and prosthesis & $39(76.5)$ & \\
\hline No speech & $4(7.8)$ & \\
\hline \multicolumn{3}{|l|}{ Speech quality ${ }^{\mathrm{a}}$} \\
\hline Good & $7(13.7)$ & \\
\hline Medium & $20(39.2)$ & \\
\hline Bad & $21(41.2)$ & \\
\hline \multicolumn{3}{|l|}{ Patient satisfaction $^{\mathrm{a}}$} \\
\hline Very satisfied & $4(7.8)$ & \\
\hline Mild complaints & $25(49.0)$ & \\
\hline Major complaints & $21(41.2)$ & \\
\hline
\end{tabular}

${ }^{a}$ Not all patients recorded speech quality and overall satisfaction solid diet, while in the free flap group this was $32.4 \%$ (Table 4).

\section{Complications}

No complication was registered in only $10 \%$ of patients during the postoperative period. $65 \%$ suffered some sort of recipient site complication, ranging from partial flap necrosis $(5.2 \%)$, wound dehiscence $(22.4 \%)$, wound infections $(24.1 \%)$, pharyngocutaneous fistulas $(36.2 \%)$ and complete flap necrosis $(12.1 \%)$. When we split up complete flap necrosis for each reconstruction type, the pectoralis major has a success rate of $87.5 \%$ (two flaps died because of anatomical vascular variation), the radial forearm $87.0 \%$ and the anterolateral thigh $90.9 \%$. Combined free flaps have a total success rate of $88.1 \%$.

$15.5 \%$ of patients had a donor site complication, where $6.9 \%$ needed re-intervention in the operating room. $31.0 \%$ had some sort of medical complication, varying from electrolyte disturbances to respiratory failure. Two patients suffered from major medical complications, leading to death. For more details, see Table 5.

Twenty-one patients $(36.2 \%)$ developed a pharyngocutaneous fistula in the postoperative period. More than half of these fistulas (11 patients, $52.4 \%$ ) were successfully treated with conservative treatment. Nine patients (42.9\%) needed flap reconstruction with a pectoralis major pedicled flap to resolve the fistula. One patient $(4.8 \%)$ could suffice with sutures for closure of the fistula (Table 6). Comparing the occurrence of fistula versus the type of surgical defect, we found no significant difference in the occurrence of fistulas (Chi-square, $p=0.664$ ).

Besides pharyngocutaneous fistulas, there were also a few problematic tracheoesophageal fistulas. These are iatrogenic fistulas, designed to hold the speech prosthesis. In total there were 53 out of 58 patients who received a TEP during, or shortly after the reconstruction surgery. Of those patients, nine $(17.0 \%)$ had a complication with the TEP. Four of these patients $(7.5 \%)$ could suffice with conservative treatment, another four needed flap surgery and one patient $(1.8 \%)$ needed additional sutures.

\section{Multivariable ordinal regression analysis}

\section{Food passage}

Multivariable ordinal regression analysis showed a significant difference between the pectoralis major flap versus radial forearm flap $(p=0.005)$ and the pectoralis major versus anterolateral thigh flap $(p=0.006)$, in favor of both free flaps. When data was transformed to compare the radial forearm and anterolateral thigh flap, no significant result was found $(p=0.365)$. Other 
Table 4 Food passage after one year in different reconstruction groups

\begin{tabular}{lrrllr}
\hline$N=51$ & Solid $n(\%)$ & Semi-solid $n(\%)$ & Fluids $n(\%)$ & GT/PEG $n(\%)$ & Total $n(\%)$ \\
\hline Pedicled flaps & & & & & \\
$\quad$ Pectoralis major & $3(17.6)$ & $6(35.3)$ & $2(11.8)$ & $6(35.3)$ & $17(100.0)$ \\
Free flaps (total) & $11(32.4)$ & $15(44.1)$ & $4(11.8)$ & $4(11.8)$ & $34(100.0)$ \\
Anterolateral thigh & $2(22.2)$ & $4(44.4)$ & $2(22.2)$ & $1(11.1)$ & $9(100.0)$ \\
Radial forearm & $9(36.0)$ & $11(44.0)$ & $2(8.0)$ & $3(4.0)$ & $25(100.0)$ \\
\hline
\end{tabular}

Table 5 Total amount of complications, categorized according to recipient site, donor site and medical complications

\begin{tabular}{llll}
\hline$N=58$ & Recipient site $n(\%)$ & Donor site $n(\%)$ & Medical $n(\%)$ \\
\hline None & $20(34.5)$ & $49(84.5)$ & $40(69.0)$ \\
Grade I & $7(12.1)$ & $4(6.9)$ & $5(8.6)$ \\
Grade II & $6(10.3)$ & 0 & $10(7.2)$ \\
Grade III & $24(41.4)$ & $5(8.6)$ & 0 \\
Grade IV & 0 & 0 & $1(1.7)$ \\
Grade V & $1(1.7)$ & 0 & $2(3.4)$ \\
\hline
\end{tabular}

significant outcomes were: circumferential surgical defect versus near-circumferential surgical defect $(p=0.004)$, in favor of the near-circumferential surgical defect. Preoperative BMI $(p=0.016)$ was also found to be significant, with an odds ratio below 1 , meaning with each point increase in BMI a higher cumulative score is more likely, suggesting a better functional outcome when BMI is high. No comorbidity versus grade II comorbidity (ACE-27 index) $(p=0.041)$ was also significant, in favor of no comorbidity. Postoperative radiation therapy did not appear to be significant in affecting the food passage $(p=0.267)$. Multivariate data with odds ratios are shown in Table 7.

\section{Complications}

In multivariable analysis for recipient site complications, both free flaps show significant less recipient site
Table 6 Pharyngocutaneous fistula per surgical defect

\begin{tabular}{lllc}
\hline & Circumferential $n=32$ & Near-circumferential $n=26$ & Total $n=58$ \\
\hline $\begin{array}{l}\text { Pharyngocutaneous fistulas } \\
\text { Intervention }\end{array}$ & $12(37.5)$ & $9(34.6)$ & $21(36.2)$ \\
Conservative treatment & $7(58.3)$ & $4(44.4)$ & $11(52.4)$ \\
Sutures & $1(8.3)$ & 0 & $1(4.8)$ \\
Flap surgery & $4(33.3)$ & $5(55.5)$ & $9(42.9)$ \\
\hline
\end{tabular}

Table 7 Multivariable ordinal regression for food passage after full recovery

\begin{tabular}{lll}
\hline Variable & Odds ratio $(95 \%$ confidence interval $)$ & $p$ value \\
\hline Type of reconstruction & & - \\
Pectoralis major & 1 (ref) & 0.005 \\
Radial forearm & $0.11(0.02-0.51)$ & 0.006 \\
Anterolateral thigh & $0.05(0.01-0.42)$ & - \\
Surgical defect & 1 (ref) & 0.004 \\
Near-circumferential & $8.58(2.00-36.78)$ & 0.016 \\
Circumferential & $0.82(0.69-0.96)$ & - \\
BMI preoperative & $1($ ref $)$ & 0.267 \\
Postoperative radiotherapy & $0.44(0.10-1.89)$ & - \\
Yes & 1 (ref) & 0.064 \\
No & $4.14(0.92-18.60)$ & 0.041 \\
ACE-27 & $8.96(1.09-73.55)$ & 0.174 \\
None & $23.59(0.25-2257.47)$ & \\
Grade I &
\end{tabular}

$p<0.05$ was considered statistically significant 
Table 8 Multivariable ordinal regression for recipient site complications

\begin{tabular}{lll}
\hline Variable & Odds ratio $(95 \%$ confidence interval) & $p$ value \\
\hline Type of reconstruction & 1 (ref) & - \\
Pectoralis major & $0.04(0.01-0.52)$ & 0.013 \\
Anterolateral thigh & $0.08(0.01-0.68)$ & 0.021 \\
Radial forearm & 1 (ref) & - \\
Surgical defect & $1.82(0.48-6.85)$ & 0.375 \\
Circumferential & 1 (ref) & - \\
Near-circumferential & $1.05(0.26-4.25)$ & 0.943 \\
Preoperative radiotherapy & $1.01(1.00-1.01)$ & 0.026 \\
Yes & 1 (ref) & - \\
No & $1.23(0.27-5.52)$ & 0.791 \\
Length of surgery & $1.23(0.18-8.67)$ & 0.832 \\
ACE-27 & $1.29(0.01-573.07)$ & 0.935 \\
None & \multicolumn{1}{l}{ Grade I } & \\
Grade II & & \\
Grade III & &
\end{tabular}

complications (radial forearm $p=0.021$, anterolateral thigh $p=0.013$ ). A longer duration of the surgery time was also found to be a significant predictor of recipient site complications $(p=0.026)$. However, in this model the type of surgical defect, preoperative radiotherapy and comorbidity were found not to be significant factors in predicting recipient site complications. Multivariate analysis and odds ratio are also shown in Table 8 . When transforming the data to compare the anterolateral thigh flap with the radial forearm flap, no significant result was found ( $p=0.209$ ).

\section{Discussion}

This study shows that hypopharyngeal reconstruction with a free radial forearm flap and free anterolateral thigh flap leads to a significantly better functional outcome, and less recipient site complication than the pectoralis major pedicled flap.

To our knowledge we are the first to use multivariable ordinal regression analysis to compare different types of reconstruction on the outcomes food passage and recipient site complications using the standardized Clavien Dindo classification system [6]. As anticipated, surgical defect (circumferential or near-circumferential) found to be a significant factor to determinate postoperative food passage. A near-circumferential defect resulted in better food passage. However, in contrast to the expectations, our results also show that the type of surgical defect does not make a difference in the formation of pharyngocutaneous fistulas. Creating two extra vertical suture lines on the posterior pharyngeal wall, does not appear to be a significant contributor to more recipient site complications, nor the formation of fistulas.
Multiple studies have demonstrated that free flaps such as the radial forearm free flap and anterolateral thigh free flap have fewer complications and the same or even better functional results regarding swallowing and speech rehabilitation when compared to a free jejunal flap [12-15]. Nevertheless, there are many articles considering the free jejunal flap as the 'golden standard' for circumferential defects. Proponents of the jejunal flap claim it has some sort of peristalsis; it is naturally tubularized and easily fits to the cranial and caudal end of the pharynx. Downsides of the jejunal flap are that it requires abdominal surgery with its additional risks, and the re-vascularized jejunal flap does not seem to withstand high dose radiotherapy. Also, jejunal flaps provide poor speech rehabilitation, as the speech tends to stay 'wet' $[16,17]$.

We found that $11 \%$ of the free flap group and $35 \%$ of the pedicled group remained dependent on a gastric tube or PEG. This is comparable with another retrospective study, including 94 patients [12], which showed $26 \%$ of patients in the pedicled group depending on a gastric tube or PEG, compared to $14 \%$ in the free flap group. Not being able to provide oneself with oral nutrition has an enormous impact on quality of life [18]. The differences between free flap and pedicled flap are even more striking in the study by Mura et al. [12]; in their free flap group $80 \%$ of patients returned to a normal unrestricted diet, compared to $0 \%$ in the pedicled flap group. In the pedicled flap group most patients $(74 \%)$ remained dependent on a semi-solid diet.

In a retrospective analysis by Benazzo et al. [13], $75 \%$ of free flap patients resumed a normal diet, whereas $25 \%$ was dependent on a soft diet. This appears inconsistent with our results, where $30 \%$ of patients with hypopharyngeal reconstructions with a free flap resumed a solid 
diet; however, in this study the term "soft diet" was not specified. In case the authors meant solid and semi-solid combined for "a normal diet", our results are roughly comparable.

In a retrospective analysis of 136 patients by Van der Putten et al. [1], different types of hypopharyngeal reconstruction were compared. They showed that $82 \%$ retained a fully oral diet without the need of gastric tube or PEG, which is also similar to our results (combined solid, semisolid and fluids is $81.4 \%$ in our results). Pharyngocutaneous fistula rate in this study was $35 \%$, which is also comparable with our results. In contrast to our findings, only $38 \%$ of laryngectomized patients had functional voice prosthesis after one year, compared to $75 \%$ in our study group.

In all other studies discussed above, it must be mentioned that jejunal transposition flaps are also included in the free flap groups, a reconstruction technique we only use with high exception in our institution. The way pharyngocutaneous fistulas are reported in literature is inconsistent. Some studies only register pharyngocutaneous fistulas where intervention is required as a complication. In contrast to this, we reported fistulas as a leakage on barium swallowing, independently of the intervention (conservative, sutures or flap surgery) that was required. A metaanalysis, published in 2008 also struggled with these inconsistencies [19]. In this meta-analysis of 20 papers about fasciocutaneous free flaps used for pharyngoesophageal reconstruction, a combined fistula rate of $13 \%$, and a stricture rate of $16 \%$ were found. This is far less than the $35 \%$ fistula rate and $37 \%$ stricture rate that we found. Overrating our fistula rate and inconsistent reporting in literature might be an explanation for the difference found.

Our overall flap failure rate was $12 \%$, with a slightly higher flap failure rate in the pedicled flap group than the free flap group (13 versus $11 \%$ ). When we compare this with other studies, combined flap failure rates varies from 0 to $9.5 \%[1,13,17,19-21]$. Pedicled pectoralis major often has a failure rate of $0 \%$, because there is no need for microvascular anastomosis [12, 13]. Instead, we found a flap failure in two patients, both due to abnormal vascular anatomy. In a few studies discussed above, patients were fitter preoperatively, had less advanced disease and less preoperative radiotherapy. This might explain the difference in flap failure rate. In our institution we have a high percentage of salvage surgery leading to impaired wound healing, and higher percentage of complications such as pharyngocutaneous fistulas or flap failure [22-24]. Despite our knowledge and generally accepted consensus that radiotherapy has a negative influence on wound healing, in our multivariable analysis this did not seem to make a significant difference. This finding is inconsistent with literature, but might be explained because more than half of our patients needed pre- and/or post-operative radiotherapy, so that this variable no longer makes a significant difference.

A prospective study should be set up to rule out selection bias in this study group. Such a study should analyze additional data, such as suturing techniques (interrupted or continuous, single loose or mattress), neck dissections (unior bilateral) and the value of an additional pectoralis major flap. The latter is suggested by some surgeons and may protect the free flap, cover exposed great vessels, minimize the risk of wound dehiscence and fistula formation [25, 26]. Besides that, a more detailed dietician report must be kept at fixed intervals postoperatively of the patient's ability to swallow, and possible complaints such as dysphagia, aspiration and reflux. Speech function and patient satisfaction should be tested objectively, for instance with the Mendelsohn's scale [27] and validated quality of life questionnaires [28] at fixed intervals.

Despite the fact that flap reconstruction of the hypopharynx after pharyngolaryngectomy remains challenging and a complex problem for head and neck surgeons, this study shows that in the majority of cases satisfactory functional results in terms of swallowing and speech rehabilitation can be achieved.

Our data shows that the pectoralis major group is more prone to get higher grades of recipient site complications, and a worse functional outcome. Both anterolateral thighand radial forearm free flap show significant better functional outcome in swallowing, and less recipient site complications. Based on these results, we conclude that free flap reconstruction of hypopharyngeal defects must remain the first choice whenever patients are fit enough. A pedicled pectoralis major flap should be reserved for cases where a free flap isn't safe or possible. A prospective, most preferably multicentre study is recommended to eliminate selection bias of patients.

\section{Compliance with ethical standards}

Conflict of interest The authors declare that they have no conflict of interest.

Ethical approval All procedures performed in studies involving human participants were in accordance with the ethical standards of the institutional and/or national research committee and with the 1964 Helsinki declaration and its later amendments or comparable ethical standards.

Informed consent This article does not contain any studies with animals performed by any of the authors.

Open Access This article is distributed under the terms of the Creative Commons Attribution 4.0 International License (http://crea tivecommons.org/licenses/by/4.0/), which permits unrestricted use, distribution, and reproduction in any medium, provided you give appropriate credit to the original author(s) and the source, provide a 
link to the Creative Commons license, and indicate if changes were made.

\section{References}

1. van der Putten L, Spasiano R, de Bree R, Bertino G, Leemans CR, Benazzo M (2012) Flap reconstruction of the hypopharynx: a defect orientated approach. Acta Otorhinolaryngol Ital 32(5):288-296

2. Gourin CG, Johnson JT (2009) A contemporary review of indications for primary surgical care of patients with squamous cell carcinoma of the head and neck. Laryngoscope 119(11):2124-2134

3. Couch ME (2002) Laryngopharyngectomy with reconstruction. Otolaryngol Clin North Am 35(5):1097-1114

4. Welkoborsky HJ, Deichmuller C, Bauer L, Hinni ML (2013) Reconstruction of large pharyngeal defects with microvascular free flaps and myocutaneous pedicled flaps. Curr Opin Otolaryngol Head Neck Surg 21(4):318-327

5. Piccirillo JF, Tierney RM, Costas I, Grove L, Spitznagel EL Jr (2004) Prognostic importance of comorbidity in a hospital-based cancer registry. JAMA 291(20):2441-2447

6. Dindo D, Demartines N, Clavien PA (2004) Classification of surgical complications: a new proposal with evaluation in a cohort of 6336 patients and results of a survey. Ann Surg 240(2):205-213

7. Clavien PA, Barkun J, de Oliveira ML, Vauthey JN, Dindo D, Schulick RD et al (2009) The Clavien-Dindo classification of surgical complications: five-year experience. Ann Surg 250(2):187-196

8. Monteiro E, Sklar MC, Eskander A, de Almeida JR, Shrime M, Gullane P et al (2014) Assessment of the Clavien-Dindo classification system for complications in head and neck surgery. Laryngoscope 124(12):2726-2731

9. Perisanidis C, Herberger B, Papadogeorgakis N, Seemann R, Eder-Czembirek C, Tamandl D et al (2012) Complications after free flap surgery: do we need a standardized classification of surgical complications? Br J Oral Maxillofac Surg 50(2):113-118

10. IBM Corp A (2013) IBM SPSS Statistics for Windows. Released 2013. IBM Corp A, NY, p 22.0

11. Abreu MNS, Siqueira AL, Caiaffa WT (2009) Ordinal logistic regression in epidemiological studies. Rev Saúde Pública 43(1):2009

12. Mura F, Bertino G, Occhini A, Mevio N, Scelsi D, Benazzo M (2012) Advanced carcinoma of the hypopharynx: functional results after circumferential pharyngolaryngectomy with flap reconstruction. Acta Otorhinolaryngol Ital 32(3):154-157

13. Benazzo M, Bertino G, Occhini A, Spasiano R, Gatti P (2006) Functional outcomes in patients reconstructed with flaps following surgery for hypopharyngeal cancer. Acta Otorhinolaryngol Ital 26(3):127-132

14. McCrory AL, Magnuson JS (2002) Free tissue transfer versus pedicled flap in head and neck reconstruction. Laryngoscope 112(12):2161-2165
15. Chepeha DB, Annich G, Pynnonen MA, Beck J, Wolf GT, Teknos TN et al (2004) Pectoralis major myocutaneous flap vs revascularized free tissue transfer: complications, gastrostomy tube dependence, and hospitalization. Arch Otolaryngol Head Neck Surg 130(2):181-186

16. Benazzo M, Bertino G, Gatti P, Alessiani M, Occhini A, Spasiano R (2007) Atypical reconstructions with free jejunum flap after circumferential pharyngolaryngectomy. Microsurgery 27(1):17-20

17. Disa JJ, Pusic AL, Hidalgo DA, Cordeiro PG (2003) Microvascular reconstruction of the hypopharynx: defect classification, treatment algorithm, and functional outcome based on 165 consecutive cases. Plast Reconstr Surg 111(2):652-660 (discussion 661-3)

18. Lin BM, Starmer HM, Gourin CG (2012) The relationship between depressive symptoms, quality of life, and swallowing function in head and neck cancer patients 1 year after definitive therapy. Laryngoscope 122(7):1518-1525

19. Murray DJ, Novak CB, Neligan PC (2008) Fasciocutaneous free flaps in pharyngolaryngo-oesophageal reconstruction: a critical review of the literature. J Plast Reconstr Aesthet Surg 61(10): 1148-1156

20. Piazza C, Taglietti V, Nicolai P (2012) Reconstructive options after total laryngectomy with subtotal or circumferential hypopharyngectomy and cervical esophagectomy. Curr Opin Otolaryngol Head Neck Surg 20(2):77-88

21. Joo YH, Sun DI, Cho KJ, Park JO, Kim MS (2011) Fasciocutaneous free flap reconstruction for squamous cell carcinoma of the hypopharynx. Eur Arch Otorhinolaryngol 268(2):289-294

22. Suzuki S, Yasunaga H, Matsui H, Horiguchi H, Fushimi K, Yamasoba T (2015) Pharyngocutaneous fistula and delay in free oral feeding after pharyngolaryngectomy for hypopharyngeal cancer. Head Neck

23. Ganly I, Patel S, Matsuo J, Singh B, Kraus D, Boyle J et al (2005) Postoperative complications of salvage total laryngectomy. Cancer 103(10):2073-2081

24. Paydarfar JA, Birkmeyer NJ (2006) Complications in head and neck surgery: a meta-analysis of postlaryngectomy pharyngocutaneous fistula. Arch Otolaryngol Head Neck Surg 132(1):67-72

25. Gras R, Bouvier C, Guelfucci B, Robert D, Giovanni A, Zanaret M (2000) The pectoralis myofascial flap in oropharyngeal and pharyngolaryngeal reconstruction in salvage surgery. Ann Otolaryngol Chir Cervicofac 117(6):378-382

26. McLean JN, Nicholas C, Duggal P, Chen A, Grist WG, Losken A et al (2012) Surgical management of pharyngocutaneous fistula after total laryngectomy. Ann Plast Surg 68(5):442-445

27. Mendelsohn M, Morris M, Gallagher R (1993) A comparative study of speech after total laryngectomy and total laryngopharyngectomy. Arch Otolaryngol Head Neck Surg 119(5):508-510

28. Perry A, Casey E, Cotton S (2015) Quality of life after total laryngectomy: functioning, psychological well-being and selfefficacy. Int J Lang Commun Disord 